\title{
Switching noise as a probe of statistics in the fractional quantum Hall effect
}

\author{
Eytan Grosfeld, ${ }^{1}$ Steven H. Simon, ${ }^{2}$ and Ady Stern ${ }^{1}$ \\ ${ }^{1}$ Department of Condensed Matter, Weizmann Institute of Science, Rehovot 76100, Israel \\ ${ }^{2}$ Bell Laboratories, Lucent Technologies, 600 Mountain Avenue, Murray Hill, New Jersey 07974
}

\begin{abstract}
We propose an experiment to probe the unconventional quantum statistics of quasi-particles in fractional quantum Hall states by measurement of current noise. The geometry we consider is that of a Hall bar where two quantum point contacts introduce two interfering amplitudes for backscattering. Thermal fluctuations of the number of quasi-particles enclosed between the two point contacts introduce current noise, which reflects the statistics of the quasi-particles. We analyze abelian $\nu=1 / q$ states and the non-abelian $\nu=5 / 2$ state.
\end{abstract}

The fractional quantum Hall effect (FQHE) is characterized by unconventional properties of its quasiparticles. In particular, the quasi-particles carry a charge which is a fraction of the electron's charge and satisfy a fractional statistics. In abelian quantum Hall states, such as the $\nu=1 / 3$ state, the winding of one quasi-particle around another is associated with a phase shift to the wave function which is a fraction of $2 \pi$. Even more interesting are non-abelian states, such as the $\nu=5 / 2$ state: when quasi-particles are present in the ground state, due to a slight deviation of the filling factor from $\nu=5 / 2$, the ground state becomes degenerate. Then, a winding of one quasi-particle around another is associated with a unitary transformation in the subspace of ground states, leading to non-abelian statistics.

Probing the statistics is difficult in both the abelian and the non-abelian cases, as one should devise experiments that are sensitive to the non-local statistical interaction between two such quasi-particles. Recent theoretical works suggest that interference experiments in a two point contact geometry may serve as probes of statistics. Such experiments were suggested in the context of the abelian FQHE 2], the non-abelian $\nu=5 / 2$ 3, 4, 5], and the non-abelian $\nu=12 / 5$ [6, 7].

The geometry considered in these experiments (see Fig. 1]) is a Hall bar with two quantum point contacts. We shall refer to the area included between these two quantum point contacts as the "island". The left and right quantum point contacts introduce weak back-scattering of quasi-particles between the two edges, characterized by tunneling amplitudes $t_{L}$ and $t_{R}$. The back-scattered current, driven by a source-drain potential $V_{s d}$, is measured. Its magnitude is determined by the interference of the two tunneling amplitudes. An oscillating interference pattern may be observed when the area of the island is varied by means of a side gate.

The fractional statistics of the FQHE quasi-particles manifests itself in the dependence of the interference pattern on the number of quasi-particles localized within the island, denoted by $n_{\text {is }}[2,3,[4,5,6,7]$. This number may be varied either by means of an anti-dot within the center of the island, or by a variation of the magnetic field. For the abelian states $(\nu=1 / q)$ the quasi-particles in- troduce a phase shift: a change of $n_{i s}$ by one leads to a phase shift of $2 \pi / q$ in the interference pattern[2]. More interestingly, for the non-abelian $\nu=5 / 2$ the interference term is turned off when $n_{\text {is }}$ is odd, and is turned on when $n_{\text {is }}$ is even [4, $[5]$. The phase of the interference term when $n_{\text {is }}$ is even is a subtle issue, to be elaborated on below. At zero temperature, and when the bulk chemical potential is within a range of localized states, the number $n_{\text {is }}$ is determined by disorder, interactions and magnetic field, and is time independent. Thermal fluctuations, however, may make that number time dependent.

In this paper we consider the effect of thermal fluctuations of $n_{\text {is }}$. We show that if the characteristic time scale of the fluctuations is long enough (though much shorter than the time of the experiment), they do not wash out effects of abelian and non-abelian statistics. We show that in this case the back-scattered current becomes time dependent, due to the time dependence of $n_{\text {is }}$. This time dependence is a source of a unique type of current noise, whose properties reflect the fractional statistics of the quasi-particles. We analyze the conditions under which this source of noise is larger than shot noise, and explain how it may be distinguished from other noise sources.

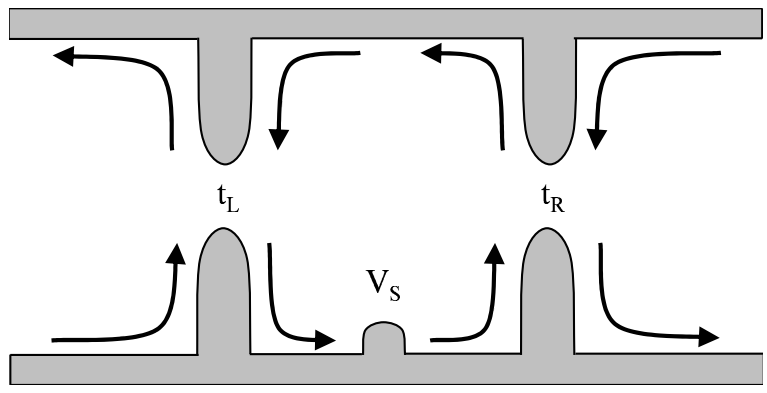

FIG. 1: Experimental setup shared by recently proposed interference experiments. Quasi-particles arrive from the left, along the lower edge, and back-scatter from the left or right quantum point contacts with tunneling amplitudes $t_{L}$ and $t_{R}$. The area of the island can be controlled by varying the voltage on a side gate.

For a given $n_{\text {is }}$ the conductance for the abelian states 
is given by [2]

$$
G=G_{0}\left[1+\beta \cos \left(\phi_{0}+2 \pi n_{\mathrm{is}} / q\right)\right]
$$

and for the $\nu=5 / 2$ state it is given by [4, 5]

$$
G= \begin{cases}G_{0} & \left(n_{\text {is }} \text { odd }\right) \\ G_{0}\left[1+\beta \cos \left(\phi_{0}+\pi n_{\text {is }} / 4+r \pi\right)\right] & \left(n_{\text {is }} \text { even }\right)\end{cases}
$$

Here $G_{0}$ is the conductance in the absence of interference $G_{0} \propto\left|t_{L}\right|^{2}+\left|t_{R}\right|^{2}$, and $\beta \propto\left|t_{R} t_{L}\right| / G_{0}$ is the visibility of the interference, both calculated to lowest order in the tunneling amplitudes. The parameter $r$ assumes the value 0 or 1 ; the probability for the two values is equal, and as long as $n_{\text {is }}$ does not vary, $r$ remains fixed as well. Its behavior when $n_{\text {is }}$ varies is studied below. The phase $\phi_{0}$ is independent of $n_{\text {is }}$ and may be controlled by changing the area of the island by means of a side gate. At finite temperatures, the conductance $G$ becomes timedependent due to hopping of quasi-particles through the quantum point contact, leading to fluctuations in $n_{\text {is }}$. Measurement of the conductance would reveal these fluctuations. If the hopping events are slow compared with the measurement time, one could observe single hopping events, manifested as abrupt changes of the conductance between the allowed values prescribed by Eqs. (1) and (2) giving clear evidence of the statistics. However, if the measurement time is larger than the hopping time, the measured conductance would approximately average Eqs. (11),(2), while the fluctuations in $n_{\text {is }}$ would result in current noise. We now proceed to analyze this noise, and show that valuable information about the statistics of the quasi-particles remains encoded into that noise.

We consider two scenarios for the fluctuations of $n_{\text {is }}$. In the first, a single quasi-particle hops between two trapping centers located on the two sides of the tunneling point of one of the quantum point contacts. Consequently, $n_{\text {is }}$ fluctuates between two consecutive integers, $n_{0}$ and $n_{0}+1$. For abelian states, the conductance fluctuates between two values, and the noise reflects these fluctuations. For non-abelian states, $n_{\text {is }}$ fluctuates between an even and an odd number, and the interference term is turned on and off by the fluctuations. Remarkably, in this case we find the noise to depend on the parity of $n_{0}$.

The second scenario is that in which the fluctuations in $n_{\text {is }}$ are much larger than one. Surprisingly, even this limit holds interesting consequences for the measured noise, both for $\nu=1 / q$ and $\nu=5 / 2$.

In all the cases we consider the system has several possible values for the conductance. Quasi-particle hopping leads to random and abrupt switches of the conductance between the allowed values, that result in telegraph-type noise in the measured conductance $G(t)$ (note that we define the conductance here as the ratio of the back-reflected current to $\left.V_{d s}\right)$. We assume certain rules and transition rates for the hopping of quasi-particles, and com- pute the current noise by calculating the two-time correlation function $S_{2}(t)=\langle\tilde{G}(t) \tilde{G}(0)\rangle V_{s d}^{2}$ and the three-time correlation function $S_{3}\left(t, t^{\prime}\right)=\left\langle\tilde{G}\left(t^{\prime}\right) \tilde{G}(t) \tilde{G}(0)\right\rangle V_{s d}^{3}$ where $\tilde{G}(t)=G(t)-\langle G\rangle$ and $\langle G\rangle$ is the average conductance. We transform to frequency space using

$$
\begin{aligned}
S_{2}(\omega) & =\int d t e^{i \omega t} S_{2}(t) \\
S_{3}\left(\omega, \omega^{\prime}\right) & =\int d t d t^{\prime} e^{i \omega\left(t^{\prime}-t\right)} e^{i \omega^{\prime}\left(t+t^{\prime}\right) / 2} S_{3}\left(t, t^{\prime}\right)
\end{aligned}
$$

We first disregard the contribution of other sources of noise to the correlators. Those are discussed towards the end of the paper.

We calculate these current correlators by standard methods for Markov chains. The states of the model are labelled $1,2, \ldots$ with conductances $G_{1}, G_{2}, \ldots$ and corresponding probabilities $P_{1}, P_{2}, \ldots$. We characterize the process by a diagram whose vertices are the states while the edges, which are directed and weighted, describe allowed transitions with corresponding rates. This diagram is encoded in the transition rate matrix $M$, whose element $M_{i j}$ describes the rate of transitions between states $i$ and $j$. The $M$-matrix has two defining properties: It is conservative $M_{i i}=-\sum_{j \neq i} M_{i j}$, and $\mathbf{P}_{0}=\left(P_{1}, P_{2}, \ldots\right)^{\text {tr }}$ satisfies $M \mathbf{P}_{0}=0$. Given that we start in state $j$ at time $t=0$, the probability $P_{i j}(t)=P\left(G(t)=G_{i} \mid G(0)=G_{j}\right)$ that we end in state $i$ at time $t$, is given by the matrix equation

$$
\partial_{t} P=M P
$$

Its solution is $P_{i j}(t)=\left\langle i\left|e^{M t}\right| j\right\rangle$, where $|i\rangle$ is a unit vector whose $i$ 'th entry is 1 . The conditional probabilities $P_{i j}$ satisfy $P_{i j}(0)=\delta_{i j}$ and, for all $j, P_{i j}(t \rightarrow \infty)=P_{i}$. Consequently, for $t^{\prime}>t>0$, the correlators $S_{2}$ and $S_{3}$ may be written as

$$
\begin{aligned}
S_{2}(t) & =V_{s d}^{2} \sum_{i j} \tilde{G}_{i}\left\langle i\left|e^{M t}\right| j\right\rangle\left\langle j \mid \mathbf{P}_{0}\right\rangle \tilde{G}_{j} \\
S_{3}\left(t, t^{\prime}\right) & =V_{s d}^{3} \sum_{i j l} \tilde{G}_{i}\left\langle i\left|e^{M\left(t^{\prime}-t\right)}\right| j\right\rangle \tilde{G}_{j}\left\langle j\left|e^{M t}\right| l\right\rangle\left\langle l \mid \mathbf{P}_{0}\right\rangle \tilde{G}_{l}
\end{aligned}
$$

where $\left\langle i \mid \mathbf{P}_{0}\right\rangle=P_{i}$, and $\tilde{G}_{i}=G_{i}-\sum_{i} P_{i} G_{i}$ is the conductance measured with respect to the average conductance.

For the case when thermal fluctuations make a single quasi-particle hop in and out of the island near the point contact, we assume hopping events to be uncorrelated, with hopping rates $\alpha P_{\text {in }}$ and $\alpha P_{\text {out }}$ into and out of the island respectively. The probability for the quasi-particle to be inside the island is $P_{\text {in }}$, and for it to be outside the island is $P_{\text {out }}=1-P_{\text {in }}$.

We start with the $\nu=1 / q$ state, for which the two conductance values are $G_{\text {out }}$ with probability $P_{\text {out }}$ and $G_{\text {in }}$ with probability $P_{\text {in }}$. The transition rates for this case are depicted in Fig. [2]]. The correlation functions 
(a)

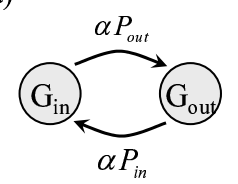

(c)

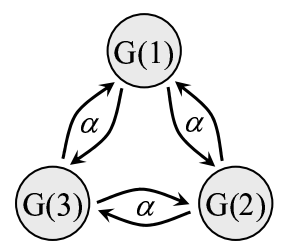

(b)

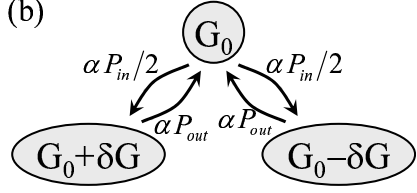

(d)

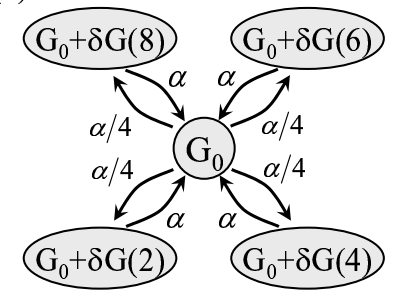

FIG. 2: The state space of the Markov chains considered in the text. (a) The abelian FQHE with one hopping quasiparticle, and the non-abelian state with $n_{0}$ even. (b) The non-abelian state with $n_{0}$ odd. (c) The abelian $\nu=1 / 3$ with a flow of quasi-particles in and out of the island. (d) The non-abelian case with a flow of quasi-particles.

are given by

$$
\begin{aligned}
S_{2}^{a}(\omega) & =\frac{2 \alpha P_{\text {in }}\left(1-P_{\text {in }}\right) \delta G^{2} V_{s d}^{2}}{\alpha^{2}+\omega^{2}} \\
S_{3}^{a}\left(\omega, \omega^{\prime}\right) & =P_{\text {in }}\left(1-P_{\text {in }}\right)\left(1-2 P_{\text {in }}\right) f\left(\omega, \omega^{\prime}\right) \delta G^{3} V_{s d}^{3}(8)
\end{aligned}
$$

where $\delta G=G_{\text {in }}-G_{\text {out }}$, and

$$
f\left(\omega, \omega^{\prime}\right)=\frac{\alpha^{2}\left(4 \omega^{2}+3{\omega^{\prime}}^{2}+12 \alpha^{2}\right)}{2\left(\omega^{\prime 2}+\alpha^{2}\right)\left(\left(\omega+\frac{\omega^{\prime}}{2}\right)^{2}+\alpha^{2}\right)\left(\left(\omega-\frac{\omega^{\prime}}{2}\right)^{2}+\alpha^{2}\right)}
$$

When compared to shot noise, where $S_{2}(\omega=0)=$ $2 e^{*}\left(P_{\text {in }} G_{\text {in }}+P_{\text {out }} G_{\text {out }}\right) V_{s d}$ and $S_{3}=0$, we see that if $P_{\text {in }} \approx 1 / 2$ and the visibility $\beta$ is close to unity, the contribution (17) typically becomes larger than the shot noise once the rate $\alpha$ is smaller than the rate at which quasiparticles cross the device, $G_{0} V / e^{*}$.

For $\nu=5 / 2$ we find a surprising dependence of the noise on the parity of $n_{0}$. This dependence originates from the subtle way in which the phase of the interference term is determined when $n_{\text {is }}$ is even. In that case there are two possible interference patterns, mutually shifted by a phase of $\pi$, corresponding to the two possible values of $r$ in Eq. (2).

We now analyze the way this parameter varies when $n_{\text {is }}$ fluctuates by the hopping of one quasi-particle. We show that when $n_{0}$ is even, $r$ remains fixed for the duration of the experiment, such that the fluctuations of $n_{\text {is }}$ translates to a conductance that switches between two possible values, with transition rates depicted in Fig. [2]. Then, the second and third cumulants of the conductance are given by Eqs. (7) and (8) with $\delta G=G_{0} \beta \cos \phi$.

In contrast, when $n_{0}$ is odd the hopping of the quasiparticle randomizes $r$, and thus translates to a switching

of the conductance between three possible values: one value, with a probability $P_{\text {in }}$, is $G_{0}$. The other two values, with equal probabilities $P_{\text {out }} / 2$, are $G_{0}[1 \pm \beta \cos (\phi)]$. The transition rates are depicted in Fig. [2]], yielding

$$
\begin{aligned}
& S_{2}^{b}(\omega)=\frac{2 \alpha P_{\text {in }} \delta G^{2}}{\left(1-P_{\text {in }}\right)^{2} \alpha^{2}+\omega^{2}} \\
& S_{3}^{b}\left(\omega, \omega^{\prime}\right)=0
\end{aligned}
$$

The dependence of the conductance on the parameter $r$ reflects the degeneracy of the ground state in the presence of the $n_{\text {is }}$ localized quasi-particles, a degeneracy which is the cornerstone of non-abelian statistics. We now review that dependence shortly $3,4,5]$, before analyzing the way it is affected by fluctuations in $n_{\text {is }}$.

The $\nu=5 / 2$ state may be described as a $p$-wave superconductor of composite fermions [8], with the quasiparticles being vortices in that super-conductor. Each vortex carries a localized zero energy core state, which is a Majorana fermion $\gamma$, satisfying $\gamma^{\dagger}=\gamma$, and $\gamma^{2}=1$. The zero energy core-states make the ground state degenerate. Mutual winding of vortices induces a transformation within the subspace of degenerate ground states. As explained in [4], when a quasi-particle (denoted by a subscript edge) that moves along the bottom edge of the system in Fig. [1, from $x=-\infty$, is back-reflected by the two quantum point contacts, the interference term of the left and right back-reflections is multiplied by the expectation value $\langle$ g.s. $|\Gamma|$ g.s. $\rangle$, with

$$
\Gamma \equiv \prod_{i=1}^{n_{\text {is }}}\left(\gamma_{e d g e} \gamma_{i}\right)
$$

where the $\gamma_{i}$ 's are the Majorana operators associated with the quasi-particles localized on the island, and $\gamma_{e d g e}$ is the Majorana operator of the back-reflected quasiparticle. When $n_{\text {is }}$ is even, $\Gamma$ depends only on the $n_{\text {is }}$ Majorana states localized in the island, and the expectation value is the same for all back-reflected quasi-particles. Then, the measurement of the conductance collapses the system into an eigenstate of $\Gamma[9]$. This operator has two eigenvalues and each one of them corresponds to a different value of $r$. When $n_{\text {is }}$ is odd, $\Gamma$ is different for different back-scattered quasi-particles, and the interference term averages to zero.

Let us now carefully examine how the value of $r$ is affected by the hopping of one quasi-particle into and out of the island. Consider first a system that fluctuates between an even $n_{0}$ and an odd $n_{0}+1$ quasi-particles in the island. When there are $n_{0}$ quasi-particles and the conductance is measured, the system collapses into an eigenstate of $\Gamma$. When the $n_{0}+1$ 'th quasi-particle hops in from outside the island, each following event of back-reflection would apply unitary transformations on $\mid$ g.s. $\rangle$, of the form $\gamma_{n_{0}+1} \gamma_{e d g e} \Gamma$. Since these transformations commute with $\Gamma$, the resulting state would still be an eigenstate of $\Gamma$, 
with the same eigenvalue. Thus, when the $n_{0}+1$ 'th quasi-particle hops out of the island again, the value of the parameter $r$ is left unchanged. The hopping of the quasi-particle into and out of the island switches the system between two values of the conductance, leading to a two-states Markov process.

The situation is different when $n_{0}$ is odd, and $n_{0}+1$ is even. Again, following the hopping of the $n_{0}+1$ 'th quasi-particle outside the island, unitary transformations of the form $\gamma_{n_{0}+1} \gamma_{e d g e} \Gamma$ are applied on the ground state, but this time, since $\gamma_{n_{0}+1}$ appears in $\Gamma$, we have,

$$
\left[\Gamma, \gamma_{n_{0}+1} \gamma_{e d g e} \Gamma\right] \neq 0
$$

Thus, in this case the back-reflection changes the state $\mid$ g.s. $\rangle$ into a state that is not an eigenstate of $\Gamma$, and randomizes the value of $r$. Consequently, the hopping of one quasi-particle switches the conductance between three values: when the quasi-particle is outside of the island, the conductance is $G_{0}$. When it is inside the island, the conductance assumes the values $G_{0}[1 \pm \beta \cos (\phi)]$, with equal probability.

We now turn to the second case where the fluctuations in $n_{\text {is }}$ are much larger than one. We start with $\nu=$ $1 / q$. The conductance assumes one of the values $G(m)=$ $G_{0}\left[1+\beta \cos \left(\phi_{0}+2 \pi m / q\right)\right]$, where $m=0,1, \ldots, q-1$ is $n_{\text {is }}$ modulo $q$. The system shifts between two adjacent $m$ values at equal rates. Consequently, the $M$-matrix of this problem is that of a tight-binding model with $q$ sites and periodic boundary conditions; its eigenvalues are $-(1-\cos (k))$, where $k=0,2 \pi / q, \ldots,(q-1) 2 \pi / q$. We can therefore Fourier transform and write the correlation function as

$$
S_{2}^{c}(t)=\sum_{x y, k} \tilde{G}_{x} \tilde{G}_{y} e^{i k(y-x)} e^{-\alpha t(1-\cos (k))}
$$

Consequently, we get

$$
S_{2}^{c}(\omega)=\frac{\alpha\left(\beta G_{0}\right)^{2}}{\alpha^{2}[1-\cos (2 \pi / q)]^{2}+\omega^{2}}
$$

and $S_{3}^{c}$ is zero.

For $\nu=5 / 2$ we get the following picture. When the instantaneous number of quasi-particles in the island is odd, no interference pattern occurs, and the conductance is $G_{0}$. When it is even, the state of the system collapses into one of the eigenvalues of $\Gamma$ with equal probability. The possible values of the conductance for even $n_{\text {is }}$ are $G_{0}\left[1 \pm \beta \cos \left(\phi_{0}\right)\right], G_{0}\left[1 \pm \beta \sin \left(\phi_{0}\right)\right]$, with equal probabilities. The diagram describing the Markov process consists of the odd particle number state in the middle and four branches going out to each of the even states. The resulting two-time correlation function is

$$
S_{2}^{d}(\omega)=\frac{1}{2} \frac{\alpha\left(\beta G_{0}\right)^{2}}{\alpha^{2}+\omega^{2}}
$$

Three-time correlations again vanish.
Finally, we turn to discuss other mechanisms of current noise and possible methods to differentiate between them and the noise we focus on here.

At high frequencies, the noise spectrum is typically dominated by shot noise, given by $2 e^{*}\left\langle I_{B}\right\rangle$ where $\left\langle I_{B}\right\rangle$ is the average back-reflected current. The linear dependence of shot noise on current is in sharp contrast to the $\left\langle I_{B}\right\rangle^{2}$ dependence of the noise we discuss here. Furthermore, the noise we discuss here is a consequence of interference of two amplitudes for back-reflection. Thus, it is turned off when only one point contact is active, in contrast to shot noise, which is present also for a single point contact.

At low frequencies, the noise spectrum will be dominated by $1 / f$ noise due to random motion of impurities. This type of noise may show similar characteristics to the noise we discuss here. Its existence is usually attributed to resistance changes due to activated processes with uniformly distributed activation energies over a small range [10]. However, while $1 / f$ noise is common to integer and fractional quantum Hall states, the contribution we discuss here is unique to the FQHE states. if one tunes the system to an integer filling factor, say $\nu=2$, the contribution to the noise described in this paper should disappear. All other sources of noise (save the shot noise) remain intact, and this method can be used to isolate the statistical noise unique to the fractional states.

To conclude, we showed that charge fluctuations on the island result in a novel type of noise unique to the fractional quantum Hall effect. For the non-abelian case, we found an even-odd effect for the noise spectrum which has its origins in the non-abelian properties of the quasiparticles. Consequently, the noise spectrum of the backscattered current may serve as a probe of the unique properties of the abelian FQHE states and the nonabelian $\nu=5 / 2$ state.

We acknowledge financial support from the US-Israel BSF (2002-238) and the Minerva foundation.

[1] G. Moore and N. Read, Nucl. Phys. B360, 362 (1991).

[2] C. de C. Chamon, D. E. Freed, S. A. Kivelson, et al., Phys. Rev. B55, 2331-2343 (1997)

[3] S. Das Sarma, M. Freedman, and C. Nayak, Phys. Rev. Lett. 94, 166802 (2005).

[4] A. Stern and B. Halperin, cond-mat/0508447

[5] P. Bonderson, A. Kitaev, and K. Shtengel, cond-mat/0508616

[6] P. Bonderson, K. Shtengel, J.K. Slingerland, cond-mat/0601242

[7] S.B. Chung, M.Stone, cond-mat/0601594

[8] N. Read and D. Green, Phys. Rev. B 61, 10267 (2000).

[9] B.J. Overbosch, F.A. Bais, Phys. Rev. A 64, 062107 (2001).

[10] Y. Imry, Introduction to Mesoscopic Physics, 2nd ed., Oxford, New York, 2002. 